\title{
A Fractional Model for the Allometric Scaling Laws
}

\author{
Zhengang Zhao ${ }^{1}$, Qian $\mathrm{Guo}^{2}$ and Changpin $\mathrm{Li}^{*}{ }^{1}$ \\ ${ }^{1}$ Department of Mathematics, Shanghai University, Shanghai 200444, P.R. China \\ ${ }^{2}$ Department of Mathematics, Shanghai Normal University, Shanghai 200234, P.R. China
}

\begin{abstract}
From the available literature, the allometric scaling laws generally exist in biology, ecology, etc. These scaling laws obey power law distributions. A possibly better approach to characterize the power law is to utilize fractional derivatives. In this paper, we establish a fractional differential equation model for this allometry by using the Caputo fractional derivatives.
\end{abstract}

Keywords: Biology, Allometric scaling law, Caputo fractional derivative, Fractional differential equation.

\section{INTRODUCTION}

From the literature available, the scaling laws of allometry typically appear in biology, ecology, etc [1-14]. For details, the dependance of a biological variable $Y$ upon body mass $M$ is emblematically characterized by an allometric scaling law (namely, power law) of the form

$Y=Y_{0} M^{\alpha}$

where $\alpha$ is a scaling exponent and $Y_{0}$ is a constant that is determined by the kind of organism.

In [1], West et al. proposed that a common mechanism underlies following laws: Living things are sustained by the transport of materials through linear networks that branch to supply all parts of the organism. Under this mechanism axiom, they established a quantitative model that explains the origin and ubiquity of quarter-power scaling and also predicts the essential feature of transport systems, such as mammalian blood vessels, bronchial trees, plant vascular systems and insect tracheal tubes. Their model was derived on the basis of three postulates: (i) a space-filling network that branches hierarchically to supply all parts of the threedimensional body; (ii) body-size invariant terminal units, such as capillaries or leaf petioles; and (iii) minimization of the energy and time required to distribute resources.

The ubiquitous scaling law (1) commonly exists in nature, but for living things, the scaling factors $\alpha$ behave multiplicity of quarter. For example, metabolic rates $B$ of entire organisms scales as $M^{3 / 4}$, that is, $B \propto M^{3 / 4}$; rates of cellular metabolism, heartbeat and maximal population growth scale as $M^{-1 / 4}$; and times of blood circulation, embryonic growth and development, and life-span scale as $M^{1 / 4}$ [1-14]. The " $3 / 4$ scaling property" was specifically and detailed reported for mature seed plants [9]. In those papers, a very

*Address correspondence to this author at the Department of Mathematics, Shanghai University, Shanghai 200444, P. R. China;

E-mail: 1cp@shu.edu.cn interesting fact is shown: the mass of dry leaves $M_{L}$ scales to the $3 / 4$ power of the mass of dry root $M_{R}$, the mass of dry leaves $M_{L}$ scales to the $3 / 4$ power of the mass of dry stem $M_{S}$, which follows that $M_{S} \propto M_{R}$. The perfect quarter-power scaling law well reflects life phenomena to some extent [15]. Besides, for early plant ontogeny, a different allometric scaling law was presented by [16]. Allometric scaling law is characterized by fractional power, which lies in the fractal world [17]. One of useful tools which are used to disclose fractal is the fractional calculus [18-21]. In this paper, we derive a fractional differential equation model for the allometry of scaling laws by using the Caputo fractional derivative [19, 21-30].

\section{FRACTIONAL EQUATION MODEL}

Before deriving a fractional equation model for allometry of scaling laws, we first introduce several fundamental definitions of fractional calculus.

In general, four fractional derivative definitions, i.e., Grünwald-Letnikov fractional derivative, Riemann-Liouville fractional derivative, generalized fractional derivative in the sense of generalized functions, and Caputo's fractional derivative, are mostly used [19, 21-24, 31-34]. The former three definitions are often used by pure mathematicians while the last one is adopted by applied scientists since it is more convenient in engineering applications. Here we only discuss Caputo derivative:

$$
{ }_{C} D_{0, t}^{q} x(t)=J^{m-q} x^{(m)}(t), q>0,
$$

Where $m=\lceil q\rceil$, i.e., $m$ is the first integer which is not less than $q, x^{(m)}$ is a conventional $m$-th order derivative, $J^{\beta}$ is the $\beta$-th order Riemann-Liouville integral operator which is expressed as follows:

$$
J^{\beta} x(t)=\frac{1}{\Gamma(\beta)} \int_{0}^{t}(t-\tau)^{\beta-1} x(\tau) d \tau, \beta>0
$$


in which $\Gamma(\beta)$ is the usual Euler function, i.e., $\Gamma(\beta)=\int_{0}^{+\infty} e^{-t} t^{\beta-1} d t$

Although these three fractional derivatives with the same order $q>0$ are not equivalent, the Grünwald-Letnikov fractional integral ${ }_{G L} D_{0, t}^{-q}$, the Riemann-Liouville fractional integral ${ }_{R L} D_{0, t}^{-q}$, and the Caputo's fractional integral ${ }_{C} D_{0, t}^{-q}$, with order $q>0$ have the same expression. Obviously, $J^{\beta}$ and ${ }_{R L} D_{0, t}^{-\beta}$ are the same. In order to coincide with (2), here and throughout, denote $J^{\beta}$ by $_{C} D_{0, t}^{-\beta}, \beta>0$.

Caputo derivative (integral) has following properties:

(i) ${ }_{C} D_{0, t}^{\alpha} t^{\gamma}=\frac{\Gamma(\gamma+1)}{\Gamma(-\alpha+\gamma+1)} t^{\gamma-\alpha}$

holds for $\alpha<0, \gamma>-1$ or $0 \leq m \leq \alpha<m+1, \gamma>m \in Z^{+}$;

(ii) ${ }_{C} D_{0, t}^{\alpha} d=0$

for any constant $d$ and $\alpha>0$.

(iii) The Caputo differential operator is a linear operator, i.e.

$$
\begin{gathered}
{ }_{C} D_{0, t}^{\alpha}(a x+b y)(t)=a \cdot{ }_{C} D_{0, t}^{\alpha} x(t) \\
+b \cdot{ }_{C} D_{0, t}^{\alpha} y(t) .
\end{gathered}
$$

for arbitrary constants $a$ and $b$;

(iv) ${ }_{C} D_{0, t}^{\alpha} \cdot{ }_{C} D_{0, t}^{\beta}={ }_{C} D_{0, t}^{\beta} \cdot{ }_{C} D_{0, t}^{\alpha}={ }_{C} D_{0, t}^{\alpha+\beta}$

holds for $\alpha \cdot \beta>0$ and $0<\alpha+\beta \leq 1$ [31], or $\alpha<0$ and $\beta<0$.

On the other hand, the fractional differential equation

${ }_{C} D_{0, t}^{\alpha} y(t)=f(t)$

with initial value condition

$y^{(k)}(0)=y_{0}^{k}, k=0,1, \cdots,\lceil\alpha\rceil-1$,

is equivalent to a Volterra integral equation

$y(t)=\sum_{k=0}^{\lceil\alpha-1} y_{0}^{k} \frac{t^{k}}{k !}+\frac{1}{\Gamma(\alpha)} \int_{0}^{t}(t-\tau)^{\alpha-1} f(\tau) d \tau$.

Next, we establish a fractional model. Here data come from the Appendix of one paper [12,35] (the web page can be found from the reference of this paper).

Let also $B$ denotes metabolic rates, $M$ total plant biomass; $M_{L}$ the mass of dry leaves, $M_{S}$ the mass of dry stems; $M_{L}$ the mass of dry leaves, $M_{R}$ the mass of dry roots. Assume still $B$ and $M, M_{L}$

and $M_{S}, M_{L}$ and $M_{R}$ satisfy the following relations,

$B=a_{0} M^{\alpha}, M_{L}=b_{0} M_{S}^{\beta}, M_{L}=c_{0} M_{R}^{\gamma}$

By the method of least square, we find that $B=0.0158 M^{0.7595}, M_{L}=0.9642 M_{S}^{0.7486}$,
$M_{L}=1.4543 M_{R}^{0.7413}$,

where the node number are 344,460 and 171 respectively, see Appendix in which $a_{1}=0$. These relations are in line with prediction, $B \propto M^{0.75}, M_{L} \propto M_{S}^{0.75}, M_{L} \propto M_{R}^{0.75}$.

Their diagrams are in Fig. (1), which is almost the same with that in Enquist-Niklas's work [9].

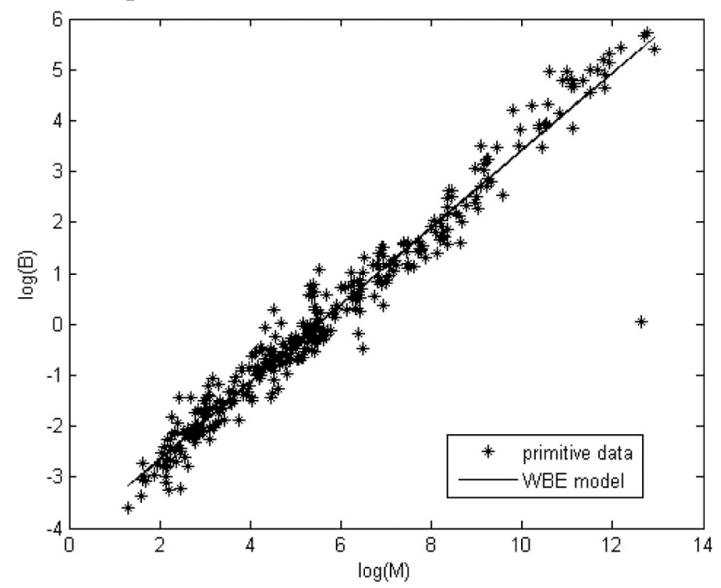

(a)

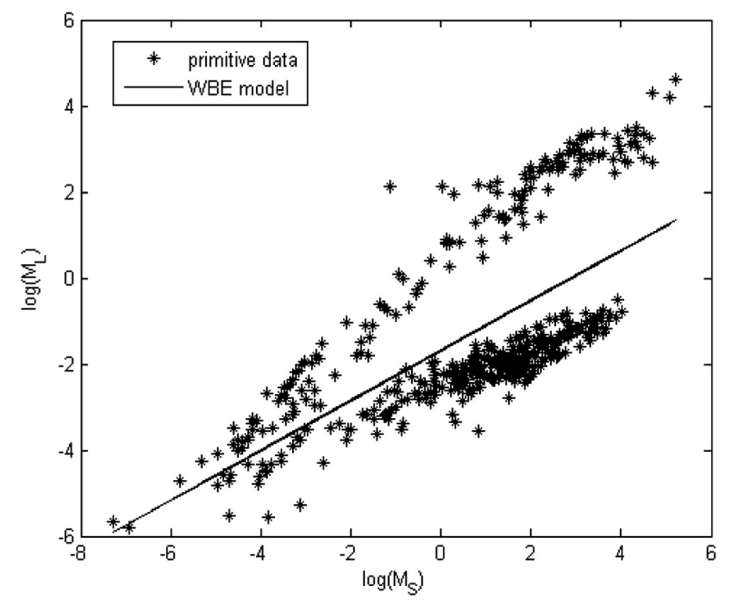

(b)

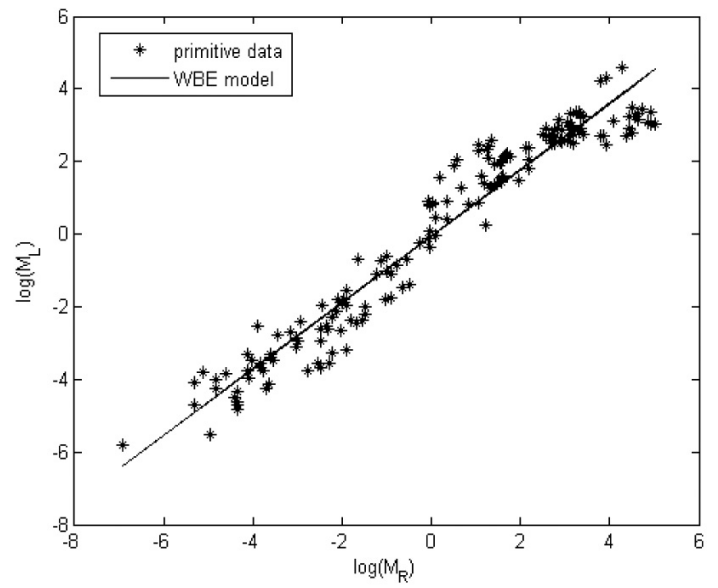

(c)

Fig. (1). The log-log linear curve denotes the statistical fit of actual data (original units in $\mathrm{kg}$ of dry weight per plant) WBE stands for West-Brown-Enquist model. (a) $\log (B)$ versus $\log (M)$; (b) $\log \left(M_{L}\right)$ versus $\log \left(M_{S}\right)$; (c) $\log \left(M_{L}\right)$ versus $\log \left(M_{R}\right)$. 
We also verify a relation between $M_{S}$ and $M_{R}$ by the same numerical method,

$M_{S} \propto M_{R}$,

in which the node number is 171 .

It is easy to see that three equations of (4) satisfy the following equations, respectively,

$$
\begin{gathered}
\left\{\begin{array}{c}
{ }_{C} D_{0, M}^{0.7595} B(M)=0.0158 \cdot \Gamma(1.7595), \\
B(0)=0 ;
\end{array}\right. \\
\left\{\begin{array}{c}
{ }_{C} D_{0, M_{S}}^{0.746} M_{L}\left(M_{S}\right)=0.9642 \cdot \Gamma(1.7486), \\
M_{L}(0)=0 ;
\end{array}\right. \\
\left\{\begin{array}{c}
{ }_{C} D_{0, M_{R}}^{0.7413} M_{L}\left(M_{R}\right)=1.4543 \cdot \Gamma(1.7413), \\
M_{L}(0)=0 .
\end{array}\right.
\end{gathered}
$$

The right hand sides of equations (5)-(7) are constants. If we assume that the relations between $B$ and $M, M_{L}$ and $M_{S}, M_{L}$ and $M_{R}$ obey the following law,

$$
\left\{\begin{array}{c}
{ }_{C} D_{0, t}^{\alpha} x(t)=f(t), \alpha \in(0,1), \\
x(0)=0
\end{array}\right.
$$

in which $f(t)=\Gamma(\alpha+2)\left(a_{0}+a_{1} t\right)$, it will be somewhat appropriate. Furthermore, one can suppose that

$f(t)=\Gamma(\alpha+m+1) \sum_{l=0}^{m} a_{l} t^{l}$.

For case with $f(t)=\Gamma(\alpha+2)\left(a_{0}+a_{1} t\right)$, (8) can be analytically solved, i.e.,

$x(t)=a_{0}(\alpha+1) t^{\alpha}+a_{1} t^{\alpha+1}$.

For the case with

$f(t)=\Gamma(\alpha+m+1) \sum_{l=0}^{m} a_{l} t^{l}$,

the solution to (8) is given by

$x(t)=\sum_{l=0}^{m} \frac{\Gamma(\alpha+m+1)}{\Gamma(\alpha+l+1)} a_{l} t^{l}$.

The existed results coincide with (10) for

$a_{1}=a_{2}=\cdots=a_{m}=0$ in reality. However, (10) is a little tedious if $m \geq 2$. In this paper, we only find unknowns $\alpha, a_{0}$ and $a_{1}$ in (9) by the method of least square, then the associate fractional model (8) follows. For details, see the Appendix.

The comparison among the primitive data, WBE model (West-Brown-Enquist model) and fractional model are in Fig. (2). From this figure, our fractional model is more appropriate.

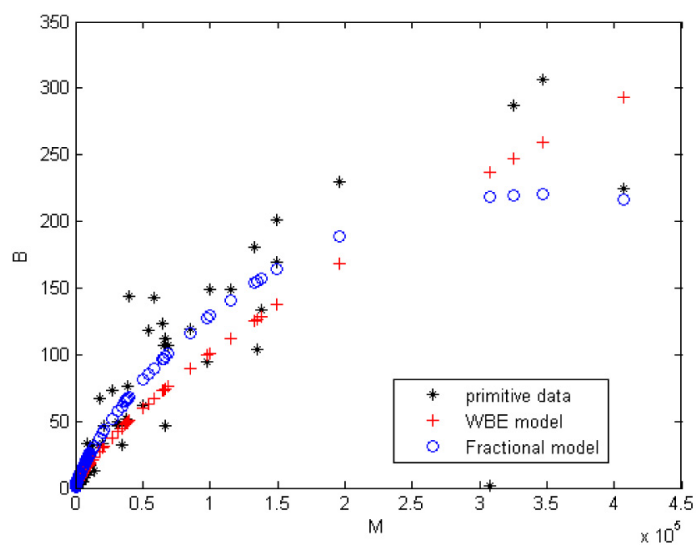

(a)

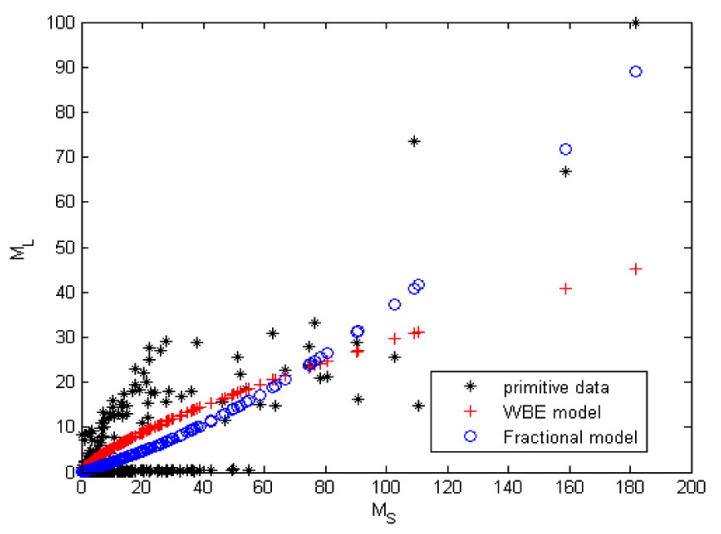

(b)

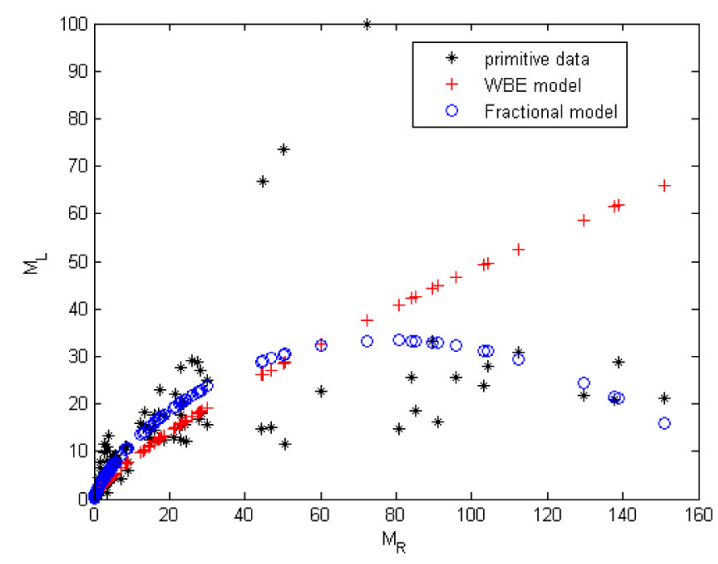

(c)

Fig. (2). The comparison between WBE and fractional model which are drawn from (5')-(7'). (a) $B$ versus $M$; (b) $M_{L}$ versus $M_{S} ;$ (c) $M_{L}$ versus $M_{R}$.

\section{CONCLUSION}

In this paper, we establish a fractional model for the allometric scaling laws in biology, ecology. The derived model works well. On one hand, if the scaling exponent $\alpha$ is negative, we can also establish an integral equation model by using the fractional derivatives. On the other hand, scaling laws widely exist in the world, which belong a fractal com- 
munity, for example, besides mentioned in biology, ecology, also in fluid, finance, complex networks, etc. To disclose the allometric secrets in fractal community, modeling the allometry is a long-term duty.

\section{APPENDIX}

To seek parameters $\alpha, a_{0}$ and $a_{1}$ of equation (9), we use the method of least square. Set

$Y=\sum_{l=0}^{n}\left[x_{i}-(\alpha+1) a_{0} t_{i}^{\alpha}-a_{1} t_{i}^{\alpha+1}\right]^{2}$

By the method of least square, one gets

$$
\left\{\begin{array}{c}
\frac{\partial Y}{\partial a_{0}}=2 \sum_{i=0}^{n}\left(x_{i}-(\alpha+1) a_{0} t_{i}^{\alpha+1}-a_{1} t_{i}^{\alpha+1}\right) \\
\cdot(\alpha+1) t_{i}^{\alpha}=0, \\
\frac{\partial Y}{\partial a_{1}}=2 \sum_{i=0}^{n}\left(x_{i}-(\alpha+1) a_{0} t_{i}^{\alpha+1}-a_{1} t_{i}^{\alpha+1}\right) \\
\cdot t_{i}^{\alpha+1}=0, \\
\frac{\partial Y}{\partial \alpha}=2 \sum_{i=0}^{n}\left(x_{i}-(\alpha+1) a_{0} t_{i}^{\alpha+1}-a_{1} t_{i}^{\alpha+1}\right) \\
\cdot\left(-a_{0} t_{i}^{\alpha}-(\alpha+1) a_{0} t_{i}^{\alpha} \ln t_{i}-a_{1} t_{i}^{\alpha+1} \ln t_{i}\right)=0 .
\end{array}\right.
$$

The system of equations (12) is nonlinear. We here use the Newton method. The results are listed below,

$$
\begin{aligned}
& B=0.0182 M^{0.7816}-2.28 \times 10^{-8} M^{1.7816}, \\
& M_{L}=0.3425 M_{S}^{0.7889}+0.00606 M_{S}^{1.7889}, \\
& M_{L}=2.1269 M_{R}^{0.7621}-0.011 M_{R}^{1.7621},
\end{aligned}
$$

They are just the solutions of following equations, respectively,

$$
\begin{gathered}
\left\{\begin{array}{c}
{ }_{C} D_{0, M}^{0.7816} B(M)=\Gamma(2.7816) \\
\cdot\left(0.0182-2.28 \times 10^{-8} M\right), \\
B(0)=0
\end{array}\right. \\
\left\{\begin{array}{c}
{ }_{C} D_{0, M_{S}}^{0.789} M_{L}\left(M_{S}\right)=\Gamma(2.7889) \\
\cdot\left(0.3425-0.00606 M_{S}\right), \\
M_{L}(0)=0 ;
\end{array}\right. \\
\left\{\begin{array}{c}
{ }_{C} D_{0, M_{R}}^{0.7413} M_{L}\left(M_{R}\right)=\Gamma(2.27621) \\
\cdot\left(2.1269-0.011 M_{R}\right), \\
M_{L}(0)=0 .
\end{array}\right.
\end{gathered}
$$

\section{REFERENCES}

[1] West GB, Brown JH and Enquist BJ. A general model for the origin of allometric scaling laws in Biology. Science 1997; 276: 12226.

[2] Beuchat CA, Kuikka J, Brown JH, Enquist BJ and West GB. Allometric scaling laws in Biology. Science 1997; 278: 369c.

[3] Kurz H, Sandau K, Dawson TH, Brown JH, Enquist BJ and West GB. Allometric scaling laws in Biology. Science 1998; 281: 751a.
[4] Enquist BJ, Brown JH and West GB. Allometric scaling of plant energetics and population density. Nature 1998; 395: 163-65.

[5] Dewar RC, Magnani F, Enquist BJ, Brown JH and West GB. Plant energetics and population density. Nature 1999; 398: 572-73.

[6] West GB, Brown JH and Enquist BJ. The fourth dimension of life: fractal geometry and allometric scaling of organisms. Science 199a; 284: 1677- 79.

[7] West GB, Brown JH and Enquist BJ. A general model for structure and allometry of plant vascular systems. Nature 1999b; 400: 66467.

[8] West GB, Savage VM, Gillooly J and Enquist BJ. Why does metabolic rate scale with body size? Nature 2003 421: 713-14.

[9] Enquist BJ and Niklas KJ. Global allocation rules for pattern of biomass partitioning in seed plants. Science 2002; 295: 1517-20.

[10] Sack L, Marańón T, Grubb PJ, Enquist BJ and Niklas KJ. Global allocation rules for patterns of biomass partitioning. Science 2002; 296: 1923a-24.

[11] Enquist BJ. Cope's rule and the evolution of long-distance transport in vascular plants: allometric scaling, biomass partitioning and optimization. Plant Cell Environ 2003; 26: 151-61.

[12] Savage VM, Gillooly JF, Woodruff WH, et al. The predominance of quarter-power scaling in Biology. Funct Ecol 2004; 18: 257.

[13] McCarthy MC and Enquist BJ. Organismal size, metabolism and the evolution of complexity in metazoans. Evol Ecol Res 2005; 7 : 681-96.

[14] Gillooly JF, Brown JH, West GB, Savage VM and Charnov EL. Effect of size and temperature on metabolic rate. Science 2001; 293: 2248-51.

[15] Brown JH, West GB, Enquist BJ. Yes, West, Brown and Enquist's model of allometric scaling is both mathematically correct and biologically relevant. Funct Ecol 2005; 19: 735-38.

[16] He JH. The allometry of leaf form in early plant ontogeny. Bull Math Biol 2005; 57: 1333-37.

[17] Williams N. Fractal geometry gets the measure of life's scales. Science 1997; 276: 34.

[18] Miller KS and Ross B. An Introduction to the Fractional Calculus and Fractional Differential Equations, John Wiley \& Sons, Inc., New York, 1993

[19] Podlubny I, Fractional Differential Equation, Academic Press, New York, 1999.

[20] West BJ, Bologna M and Grigolini P. Physics of Fractal Operators, Springer, New York, 2003.

[21] Li CP, Deng WH and Chen G. Scaling attractors of fractional differential systems. Fractals 2006;14: 303-13.

[22] Deng WH, Li CP and Guo Q. Analysis of fractional differential equation with multi-order. Fractals 2007; 15: 173-82.

[23] Caputo M. Linear models of dissipation whose $Q$ is almost frequency independent II. Geophys J Roy Astronom Soc 1967; 13 529-539.

[24] Li CP and Peng GJ. Chaos in Chen's system with a fractional order. Chaos Solitons Fractals 2004; 22: 443-50.

[25] Zhou TS and Li CP. Synchronization in fractional-order differential systems. Physic D 2005; 21: 111-25.

[26] Deng WH, Li CP and Lu JH. Stability analysis of linear fractional differential system with multiple time-delays. Nonlinear Dynamics 2007; 48: 409-16.

[27] Ge ZM and Hsu MY. Chaos in a generalized van der Pol system and in its fractional order system. Chaos, Solitons and Fractals 2007; 33: 1711-1745.

[28] Sheu LJ, Chen HK, Chen JH and Tam LM. Chaotic dynamics of the fractionally damped Duffing equation. Chaos Solitons Fractals 2007; 32: 1459-68.

[29] Sheu LJ, Chen HK, Chen JH, Tam LM, Chen WC, Lin KT and Kang Y. Chaos in the Newton-Leipnik system with fractional order. Chaos, Solitons and Fractals 2008; 36: 98-103.

[30] Petrás. A note on the fractional-order Chua's system. Chaos Solitons Fractals 2006.doi:10.1016/j.chaos.2006.10.054.

[31] Li CP and Deng WH. Remarks on fractional derivatives. Appl Math Comput 2007; 187: 777-84. 
[32] Hadid SB and Alshamani JG. Liapunov stability of differential equations of noninteger order. Arab J 1986; 7: 5-17.

[33] Soni RC and Singh Deepika. Certain fractional derivative formulae involving the product of a general class of polynomials and the multivariable. Proc Indian Acad Sci Math Sci 2002; 112(4): 55162 .
[34] Goyal SP, Jain RM and Gaur N. Fractional integral operators involving a product of generalized hypergeometric functions and a general class of polynomials. Indian J Pure Appl Math 1991; 22(5): 403-11.

[35] Science Online. Supplementary materials can be downloaded from Science Online at:www.sciencemag.org/cgi/content/full/295/5559/ 1517/DC1.

(C) Zhao et al.; Licensee Bentham Open.

This is an open access article distributed under the terms of the Creative Commons Attribution License (http://creativecommons.org/licenses/by/2.5/), which permits unrestrictive use, distribution, and reproduction in any medium, provided the original work is properly cited. 